\title{
Numerical Solution of the Nonlinear Klein-Gordon Equation Using Multiquadric Quasi-interpolation Scheme
}

\author{
M. Sarboland ${ }^{1, *}$, A. Aminataei ${ }^{2}$ \\ ${ }^{1}$ Department of Mathematics, Saveh Branch, Islamic Azad University, P.O. Box: 39187-366, Saveh, Iran \\ ${ }^{2}$ Department of Applied Mathematics, Faculty of Mathematics, K. N. Toosi University of Technology, P.O. Box: 16315-1618, Tehran, Iran
}

Copyright $(2015$ by authors, all rights reserved. Authors agree that this article remains permanently

open access under the terms of the Creative Commons Attribution License 4.0 International License

\begin{abstract}
This paper's purpose is to provide a numerical scheme to approximate solutions of the nonlinear Klein-Gordon equation by applying the multiquadric quasi-interpolation scheme and the integrated radial basis function network scheme. Our scheme uses $\theta$-weighted scheme for discretization of the temporal derivative and the integrated form of the multiquadric quasi-interpolation scheme for approximation of the unknown function and its spatial derivative. To confirm the accuracy and ability of the presented scheme, this scheme is applied on some test experiments and the numerical results have been compared with the exact solutions. Furthermore, it should be emphasized that with the presently available computing power, it has become possible to develop realistic mathematical models for the complicated problems in science and engineering. The mathematical description of various processes such as the nonlinear Klein-Gordon equation occurring in mathematical physics leads to a nonlinear partial differential equation. However, the mathematical model is only the first step towards the solution of the problem under consideration. The development of the well-documented, robust and reliable numerical technique for handing the mathematical model under consideration is the next step in the solution of the problem. This second step is at least as important as the first one. Therefore, the robustness, the efficiency and the reliability of the numerical technique have to be checked carefully.
\end{abstract}

Keywords Nonlinear Partial Differential Equation, Multiquadric Quasi-interpolation Scheme, Integrated Radial Basis Function Network, $\theta$-weighted Scheme, Temporal Derivative, Spatial Derivative, Problem of Mathematical Physics

2010 Mathematics Subject Classification: 35K61; 97N50; 33E99; 65M06; 35A23; 35Q51

\section{Introduction}

We consider the nonlinear Klein-Gordon equation in the following:

$$
\frac{\partial^{2} u}{\partial t^{2}}+\alpha \frac{\partial^{2} u}{\partial x^{2}}+F(u)=f(x, t), \quad x \in \Omega=[a, b] \subset \mathbb{R}, 0<t \leqslant T,
$$

with the initial conditions

$$
\begin{aligned}
& u(x, 0)=g_{1}(x), \quad x \in \Omega, \\
& \frac{\partial u}{\partial t}(x, 0)=g_{2}(x), \quad x \in \Omega,
\end{aligned}
$$

and the boundary condition

$$
u(x, t)=g(x, t), \quad x \in \partial \Omega,
$$

where $u=u(x, t)$ represents the wave displacement at position $x$ and time $t, \alpha$ is a known constant, $F(u)$ is the nonlinear force such that $\frac{\partial F}{\partial u} \geqslant 0$ and $g_{1}(x), g_{2}(x), g(x, t)$ and $f(x, t)$ are known functions. The function $F(u)$ takes many forms [16,38] such as: $\sin (u), \sin (u)+\sin (2 u), \sinh (u)+\sinh (2 u)$ and $e^{u}$ that characterizes the sine-Gordon, the double sineGordon, the double sinh-Gordon and Liouville equations, respectively. 
In the present work, the numerical approximation of the following nonlinear Klein-Gordon equation:

$$
u_{t t}+\alpha u_{x x}+\beta u+\gamma u^{k}=f(x, t),
$$

is considered wherein $k=2$ or $k=3$ and $\beta$ and $\gamma$ are known constants. In this case, the nonlinear force $F(u)$ is equal to $\beta u+\gamma u^{k}$.

The Klein-Gordon equation appears in various physical events such as the propagation of fluxions in the Josephson junctions, the motion of rigid pendula attached a stretched wire, and dislocations in crystals [21]. This equation is a relativistic version of the Schrodinger equation, which describes scalar spineless particles [3].

Many researchers have used various numerical methods to solve the nonlinear Klein-Gordon equation such as the finite difference method, the inverse scattering method, the auxiliary equation method, Backlund transformation, the Wadatitrace method, Hirota bilinear forms, the spectral method, pseudo-spectral method, the tanh-sech method, the Adomian decomposition method, the sine-cosine method, Jacobi elliptic functions and the Riccati equation expansion method $[4,11,15,34-37,41,42]$.

However, these methods usually require the generation and update of mesh in the problem domain and hence bring inconvenience during computation. In order to avoid mesh generation, meshless techniques have been attracted the attention of researchers recently. Such as the meshless methods based on radial basis functions (RBFs) is used to solve the Kortewegde Vries (KdV), sine-Gordon and Klein-Gordon equations [8-10,28-30,33].

In all these works, the solution function is decomposed into RBFs and its derivatives are then obtained through differentiation that caused the reduction in convergence rate. To avoid from this problem, researchers [2,17] introduced an integrated multiquadric (MQ) radial basis function networks scheme for the approximation of function and its derivatives. Numerical experiments and theoretical analysis indicate that for solving partial differential equations, integrated RBF (IRBF) procedure is more accurate in comparison with direct RBF (DRBF) procedure.

Unfortunately, these meshless methods for time-dependent and time-independent partial differential equations require to solve ill-conditioned linear system [1, 18-20,22-24, 26, 39]. So modified meshless schemes are developed, for instance, researchers [12, 25,31,32,45] proposed the meshless schemes to solve Burgers' equation and KdV equation by using MQ quasi-interpolation without solving a large scale linear system. In [14], a numerical method is developed to solve sine-Gordon equation by using high accuracy MQ quasi-interpolant operator $\mathcal{L}_{\mathcal{W}_{2}}$ without solving a linear system of equations at each time step. They use the second derivatives of a MQ quasi-interpolant operator $\mathcal{L}_{\mathcal{W}_{2}}$ to approximate the derivative in the spatial domain and finite difference to approximate the temporal derivative. In this paper, we apply integrated form of this method to solve the nonlinear Klein-Gordon equation.

This paper is organized as follows: In Section 2, a brief information of the MQ quasi-interpolation scheme is given. In Section 3, we present the proposed numerical scheme for the solution of nonlinear Klein-Gordon equation. Several numerical experiments are presented in Section 4, followed by a conclusion summary in Section 5.

\section{The multiquadric quasi-interpolation scheme}

MQ quasi-interpolation scheme is constructed directly from linear combination of MQ basis and approximated function. Three univariate MQ quasi-interpolations namely, $\mathcal{L}_{\mathcal{A}}, \mathcal{L}_{\mathcal{B}}$ and $\mathcal{L}_{\mathcal{C}}$ were introduced by Beatson and Powell [5]. Later, Wu and Schaback introduced univariate quasi-interpolation $\mathcal{L}_{\mathcal{D}}$ [43]. Based on Wu and Schaback's work, Jiang et al. [13] introduced a multilevel univariate quasi-interpolation $\mathcal{L}_{\mathcal{W}_{2}}$. To solve Klein-Gordon equation numerically, in this paper, we adopt the multilevel quasi-interpolation scheme $\mathcal{L}_{\mathcal{W}_{2}}$. For more information about MQ quasi-interpolant operators, see [5-7, 13,43,44]. The process of interpolation by using operator $\mathcal{L}_{\mathcal{W}_{2}}$ is as follows:

For a given region $\Omega=[a, b]$ and a finite set of scattered points

$$
a=x_{0}<x_{1}<\ldots<x_{N}=b, \quad h=\max _{1 \leqslant i \leqslant N}\left(x_{i}-x_{i-1}\right),
$$

quasi-interpolation of a univariate function $f:[a, b] \rightarrow \mathbb{R}$ can be constructed in the form:

$$
\mathcal{L}(f)=\sum_{i=0}^{N} f\left(x_{i}\right) \psi_{i}(x),
$$

where $\psi_{i}(x)$ is a linear combination of the MQs

$$
\psi_{i}(x)=\sqrt{c^{2}+\left(x-x_{i}\right)^{2}},
$$

and $c \in \mathbb{R}^{+}$is a shape parameter. Wu and Schaback's $\mathcal{L}_{\mathcal{D}}$ operator is defined as

$$
\mathcal{L}_{\mathcal{D}} f(x)=\sum_{i=0}^{N} f\left(x_{i}\right) \widetilde{\psi}_{i}(x),
$$

where

$$
\widetilde{\psi}_{0}(x)=\frac{1}{2}+\frac{\psi_{1}(x)-\left(x-x_{0}\right)}{2\left(x_{1}-x_{0}\right)}
$$




$$
\begin{aligned}
& \widetilde{\psi}_{1}(x)=\frac{\psi_{2}(x)-\psi_{1}(x)}{2\left(x_{2}-x_{1}\right)}-\frac{\psi_{1}(x)-\left(x-x_{0}\right)}{2\left(x_{1}-x_{0}\right)} \\
& \widetilde{\psi}_{i}(x)=\frac{\psi_{i+1}(x)-\psi_{i}(x)}{2\left(x_{i+1}-x_{i}\right)}-\frac{\psi_{i}(x)-\psi_{i-1}(x)}{2\left(x_{i}-x_{i-1}\right)}, \quad 2 \leqslant i \leqslant N-2, \\
& \widetilde{\psi}_{N-1}(x)=\frac{\left(x_{N}-x\right)-\psi_{N-1}(x)}{2\left(x_{N}-x_{N-1}\right)}-\frac{\psi_{N-1}(x)-\psi_{N-2}(x)}{2\left(x_{N-1}-x_{N-2}\right)}
\end{aligned}
$$

and

$$
\widetilde{\psi}_{N}(x)=\frac{1}{2}+\frac{\psi_{N-1}(x)-\left(x_{N}-x\right)}{2\left(x_{N}-x_{N-1}\right)} .
$$

Now, we pick a smaller set $\left\{x_{k_{j}}\right\}_{j=1}^{\bar{N}}$ from the given points $\left\{x_{i}\right\}_{i=0}^{N}$ where $\bar{N}$ is a positive integer satisfying $\bar{N}<N$ and $0=k_{0}<k_{1}<\ldots<k_{\bar{N}+1}=N$. Using the IMQ-RBF, the second derivative of $f(x)$ can be approximated by RBF interpolant $S_{f^{\prime \prime}}$ as

$$
S_{f^{\prime \prime}}(x)=\sum_{j=1}^{\bar{N}} \lambda_{j} \bar{\varphi}\left(\left|x-x_{k_{j}}\right|\right)
$$

where

$$
\bar{\varphi}(r)=\frac{s^{2}}{\left(s^{2}+r^{2}\right)^{3 / 2}}
$$

and $s \in \mathbb{R}^{+}$is a shape parameter. The coefficients $\left\{\lambda_{j}\right\}_{j=1}^{\bar{N}}$ are uniquely determined by the interpolation condition

$$
S_{f^{\prime \prime}}\left(x_{k_{i}}\right)=\sum_{j=1}^{\bar{N}} \lambda_{j} \bar{\varphi}\left(\left|x_{k_{i}}-x_{k_{j}}\right|\right)=f^{\prime \prime}\left(x_{k_{i}}\right), \quad 1 \leqslant i \leqslant \bar{N}
$$

At every points $x_{k_{i}}$ of the subset, $f^{\prime \prime}\left(x_{k_{i}}\right)$ is replaced by

$$
f_{x_{k_{i}}}^{\prime \prime}=\frac{2\left[\left(x_{k_{i}}-x_{k_{i-1}}\right) f\left(x_{k_{i+1}}\right)-\left(x_{k_{i+1}}-x_{k_{i-1}}\right) f\left(x_{k_{i}}\right)+\left(x_{k_{i+1}}-x_{k_{i}}\right) f\left(x_{k_{i-1}}\right)\right]}{\left(x_{k_{i}}-x_{k_{i-1}}\right)\left(x_{k_{i+1}}-x_{k_{i}}\right)\left(x_{k_{i+1}}-x_{k_{i-1}}\right)} .
$$

By using $f(x)$ and the coefficient $\lambda$ mentioned above, a function $e(x)$ is constructed in the form

$$
e(x)=f(x)-\sum_{i=1}^{\bar{N}} \lambda_{i} \sqrt{s^{2}+\left(x-x_{k_{i}}\right)^{2}} .
$$

Then the MQ quasi-interpolation operator $\mathcal{L}_{\mathcal{W}_{2}}$ by using $\mathcal{L}_{\mathcal{D}}$ defined by Eqs. (5) and (6) on the data $\left(x_{i}, e\left(x_{i}\right)\right)_{0 \leqslant i \leqslant N}$ with the shape parameter $c$ is defined as follows:

$$
\mathcal{L}_{\mathcal{W}_{2}} f(x)=\sum_{i=1}^{\bar{N}} \lambda_{i} \sqrt{s^{2}+\left(x-x_{k_{i}}\right)^{2}}+\mathcal{L}_{\mathcal{D}} e(x)
$$

The shape parameters $c$ and $s$ should not be the same constants as in Eq. (11). For more details about the properties and accuracy of $\mathcal{L}_{\mathcal{W}_{2}}$, one can see [13]. In this paper, we use the MQ quasi-interpolation operator $\mathcal{L}_{\mathcal{W}_{2}}$ with $h_{2}=2 h$.

The operator $\mathcal{L}_{\mathcal{W}_{2}}$ can be written in the compact form

$$
\mathcal{L}_{\mathcal{W}_{2}} f(x)=\sum_{i=0}^{N} f\left(x_{i}\right) \widehat{\psi}_{i}(x)
$$

wherein the basis functions $\widehat{\psi}_{i}(x)$ can be obtained by using Equations (8)-(11), i.e., see [27].

By writing operator $\mathcal{L}_{\mathcal{W}_{2}}$ in the compact form (12), we can use it in the indirect form for the numerical solution of partial differential equations. Also, by converting operator $\mathcal{L}_{\mathcal{W}_{2}}$ to the form (12), we do not require to solve a linear system of equations for getting of the coefficients $\lambda_{i}$ at each time step, see [14].

\section{The numerical method}

Now, we present the numerical scheme for solving the Klein-Gordon equation (1) by using the MQ quasi-interpolation operator $\mathcal{L}_{\mathcal{W}_{2}}$. In our scheme, $\theta$-weighted method is first employed to approximate the temporal derivative. Then, the highest order derivatives (second order in this paper) of the solution function are approximated by $\mathcal{L}_{\mathcal{W}_{2}}$ operator, and their lower 
order derivatives and the solution function are then obtained by symbolic integration. At the end, the collocation scheme is applied.

At First, we discretize the problem according to the following $\theta$-weighted scheme with time step size $\Delta t$,

$$
\frac{u^{n+1}-2 u^{n}+u^{n-1}}{(\Delta t)^{2}}+\theta\left[\alpha u_{x x}^{n+1}+\beta u^{n+1}\right]+(1-\theta)\left[\alpha u_{x x}^{n}+\beta u^{n}\right]+\gamma\left(u^{n}\right)^{k}=f^{n+1}
$$

where $u^{n}=u\left(x, t_{n}\right), u_{x x}^{n}=u_{x x}\left(x, t_{n}\right), f^{n}=f\left(x, t_{n}\right), t_{n}=n \Delta t$ and $0 \leq \theta \leq 1$.

After some arrangements, the following time discretized form of nonlinear Klein-Gordon equation is yield.

$$
\left[1+\beta \theta(\Delta t)^{2}\right] u^{n+1}+\alpha \theta(\Delta t)^{2} u_{x x}^{n+1}=\left[2-\beta(1-\theta)(\Delta t)^{2}\right] u^{n}-\alpha(1-\theta)(\Delta t)^{2} u_{x x}^{n}-\gamma(\Delta t)^{2}\left(u^{n}\right)^{k}+(\Delta t)^{2} f^{n+1}-u^{n-1} .
$$

Now, the integrated form of MQ quasi-interpolation scheme is used to approximate the solution function and its spatial derivatives. In order to at first, the highest order derivatives (second order in this paper) of the solution function, $u_{x x}^{n}$ is approximate by MQ quasi-interpolant operator $\mathcal{L}_{\mathcal{W} 2}$ on data $\left\{x_{j}\right\}_{j=1}^{N-1}$. After that, the approximations of low order derivative $u_{x}^{n}$ and solution function $u^{n}$ can be obtained by symbolic integration as follows:

$$
u^{n}(x)=\sum_{j=0}^{N} w_{j}^{n} \varphi_{j}(x), \quad u_{x}^{n}(x)=\sum_{j=0}^{N} w_{j}^{n} \dot{\varphi}_{j}(x), \quad u_{x x}^{n}(x)=\sum_{j=0}^{N} w_{j}^{n} \ddot{\varphi}_{j}(x),
$$

where

$$
\begin{array}{lll}
\varphi_{j}(x)=\iint \hat{\psi}_{j}(x) d x d x, & 1 \leqslant j \leqslant N-1, \quad \varphi_{0}(x)=x, \quad \varphi_{N}(x)=1, \\
\dot{\varphi}_{j}(x)=\int \hat{\psi}_{j}(x) d x, & 1 \leqslant j \leqslant N-1, \quad \dot{\varphi}_{0}(x)=1, \quad \dot{\varphi}_{N}(x)=0, \\
\ddot{\varphi}_{j}(x)=\hat{\psi}_{j}(x), & 1 \leqslant j \leqslant N-1, \quad \ddot{\varphi}_{0}(x)=0, \quad \ddot{\varphi}_{N}(x)=0,
\end{array}
$$

and

$$
w_{j}^{n}=u_{x x}^{n}\left(x_{j}\right), \quad 1 \leqslant j \leqslant N-1, \quad w_{0}^{n}=C_{1}, \quad w_{N}^{n}=C_{2},
$$

where $C_{1}$ and $C_{2}$ are integration constants. By substituting Eqs. (15) into Eqs. (14) and (3) and applying collocation method yield the following equations:

$$
\begin{aligned}
& {\left[1+\beta \theta(\Delta t)^{2}\right] \sum_{j=0}^{N} w_{j}^{n+1} \varphi_{i j}+\alpha \theta(\Delta t)^{2} \sum_{j=0}^{N} w_{j}^{n+1} \ddot{\varphi}_{i j}=\left[2-\beta(1-\theta)(\Delta t)^{2}\right] \sum_{j=0}^{N} w_{j}^{n} \varphi_{i j}} \\
& -\alpha(1-\theta)(\Delta t)^{2} \sum_{j=0}^{N} w_{j}^{n} \ddot{\varphi}_{i j}-\gamma(\Delta t)^{2}\left(\sum_{j=0}^{N} w_{j}^{n} \varphi_{i j}\right)^{k}+(\Delta t)^{2} f^{n+1}-\sum_{j=0}^{N} w_{j}^{n-1} \varphi_{i j}, \quad i=1, \ldots, N-1,
\end{aligned}
$$

and

$$
\sum_{j=0}^{N} w_{j}^{n+1} \varphi_{i j}=g^{n+1}\left(x_{i}\right), \quad i=0, N
$$

where $\varphi_{i j}=\varphi_{j}\left(x_{i}\right), \ddot{\varphi}_{i j}=\ddot{\varphi}_{j}\left(x_{i}\right), g^{n}\left(x_{i}\right)=g\left(x_{i}, t_{n}\right)$.

Equations (16) and (17) generate a system of $N+1$ linear equations in $N+1$ unknown parameters $w_{i}^{n+1}$. These equations can be written in matrix form

$$
\begin{aligned}
{\left[\left(1+\beta \theta(\Delta t)^{2}\right) \mathbf{A}_{d}+\mathbf{A}_{b}+\alpha \theta(\Delta t)^{2} \mathbf{D}\right] \mathbf{w}^{n+1} } & =\left[\left(2-\beta(1-\theta)(\Delta t)^{2}\right) \mathbf{A}_{d}-\alpha(1-\theta)(\Delta t)^{2} \mathbf{D}\right] \mathbf{w}^{n} \\
& -\gamma(\Delta t)^{2}\left(\mathbf{u}^{n}\right)^{k}+(\Delta t)^{2} \mathbf{F}^{n}-\mathbf{u}^{n-1}
\end{aligned}
$$

wherein

$$
\mathbf{A}_{d(i+1)(j+1)}=\varphi_{i j}, \quad \mathbf{A}_{b(i+1)(j+1)}=0, \quad \mathbf{D}_{(i+1)(j+1)}=\ddot{\varphi}_{i j},
$$

for $i=1, \ldots, N-1 ; j=0,1, \ldots, N$ and

$$
\mathbf{A}_{d(i+1)(j+1)}=0, \quad \mathbf{A}_{b(i+1)(j+1)}=\varphi_{i j}, \quad \mathbf{D}_{(i+1)(j+1)}=0,
$$

for $i=0, N ; j=0,1, \ldots, N$ and

$$
\mathbf{F}^{n}=\left[0, f^{n}\left(x_{1}\right), \ldots, f^{n}\left(x_{N-1}\right), 0\right]^{T}, \quad \mathbf{u}^{n}=\left[0, u^{n}\left(x_{1}\right), \ldots, u^{n}\left(x_{N-1}\right), 0\right]^{T} .
$$



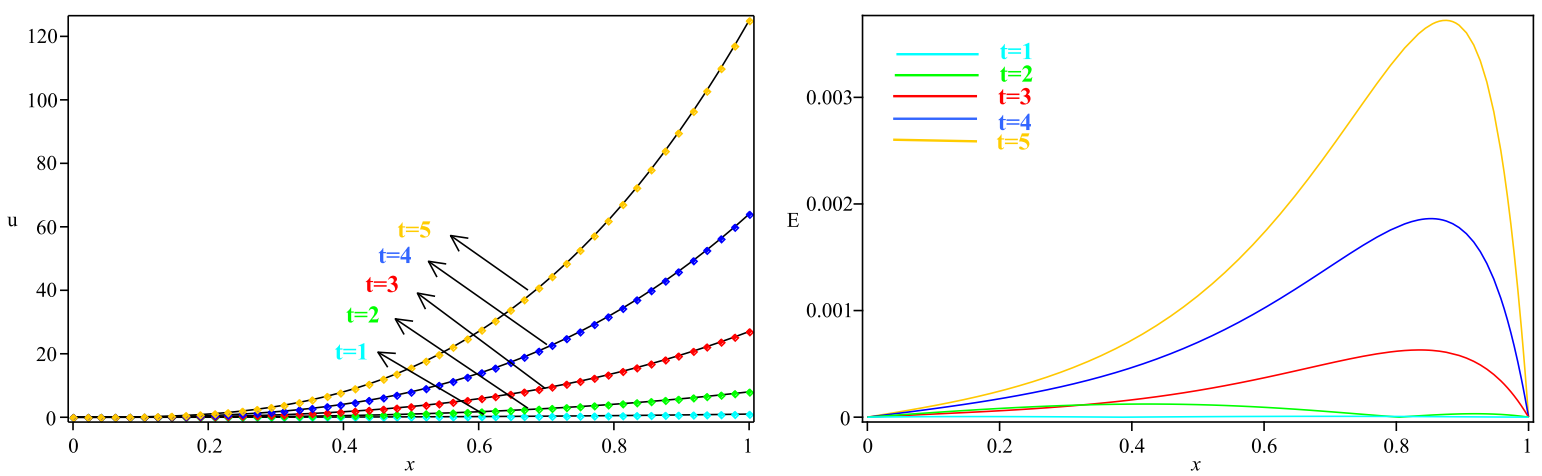

Figure 1. Exact and numerical solutions (left) and error of method (right) at different times with $\Delta t=0.0001$ and $N=20$ of experiment 1 .

Equation (18) generates a system of $N-1$ linear equations in $N-1$ unknown parameters $w_{i}^{n+1}$, which can be solved to find $\mathbf{w}^{n+1}$ in each step.

At $n=0$, Eq. (18) has the following form:

$$
\begin{aligned}
{\left[\left(1+\beta \theta(\Delta t)^{2}\right) \mathbf{A}_{d}+\mathbf{A}_{b}+\alpha \theta(\Delta t)^{2} \mathbf{D}\right] \mathbf{w}^{1}=} & {\left[\left(2-\beta(1-\theta)(\Delta t)^{2}\right) \mathbf{A}_{d}-\alpha(1-\theta)(\Delta t)^{2} \mathbf{D}\right] \mathbf{w}^{0} } \\
& -\gamma(\Delta t)^{2}\left(\mathbf{u}^{0}\right)^{k}+(\Delta t)^{2} \mathbf{F}^{0}-\mathbf{u}^{-1} .
\end{aligned}
$$

By using the second condition of Eq. (2), we have

$$
\frac{u^{1}-u^{-1}}{2 \Delta t}=g_{2}(x), \quad x \in \Omega .
$$

Substituting (20) into (19), one can get

$$
\begin{aligned}
{\left[\left(2+\beta \theta(\Delta t)^{2}\right) \mathbf{A}_{d}+\mathbf{A}_{b}+\alpha \theta(\Delta t)^{2} \mathbf{D}\right] \mathbf{w}^{1}=} & {\left[\left(2-\beta(1-\theta)(\Delta t)^{2}\right) \mathbf{A}_{d}-\alpha(1-\theta)(\Delta t)^{2} \mathbf{D}\right] \mathbf{w}^{0} } \\
& -\gamma(\Delta t)^{2}\left(\mathbf{u}^{0}\right)^{k}+(\Delta t)^{2} \mathbf{F}^{0}+2 \Delta t \mathbf{G},
\end{aligned}
$$

where $\mathbf{G}^{n}=\left[0, g_{2}\left(x_{1}\right), \ldots, g_{2}\left(x_{N-1}\right), 0\right]^{T}$.

\section{The numerical experiments}

In this section, we test the robustness and the accuracy of the presented method by four experiments. We compare our

\begin{tabular}{|c|c|c|c|c|c|c|}
\hline \multirow[t]{2}{*}{$\mathrm{t}$} & \multicolumn{3}{|c|}{ IMQQI; $N=20$} & \multicolumn{3}{|c|}{ TPSM $; N=50$} \\
\hline & $\mathrm{L}_{\infty}$ & $\mathrm{L}_{2}$ & RMS & $\mathrm{L}_{\infty}$ & $\mathrm{L}_{2}$ & RMS \\
\hline 1 & $7.7958 \mathrm{E}-06$ & $3.4694 \mathrm{E}-05$ & $4.8581 \mathrm{E}-06$ & $1.1012 \mathrm{E}-05$ & $5.4998 \mathrm{E}-05$ & $5.4725 \mathrm{E}-06$ \\
\hline 2 & $1.2307 \mathrm{E}-04$ & $5.5475 \mathrm{E}-04$ & $7.7680 \mathrm{E}-05$ & $1.6496 \mathrm{E}-04$ & $1.1522 \mathrm{E}-03$ & $1.1465 \mathrm{E}-04$ \\
\hline 3 & $5.3019 \mathrm{E}-04$ & $2.4618 \mathrm{E}-03$ & $2.4473 \mathrm{E}-04$ & $5.9728 \mathrm{E}-04$ & $3.2588 \mathrm{E}-03$ & $3.2426 \mathrm{E}-04$ \\
\hline 4 & $1.8601 \mathrm{E}-03$ & $7.1623 \mathrm{E}-03$ & $9.7029 \mathrm{E}-04$ & $1.8264 \mathrm{E}-03$ & 9.8191E-03 & $9.7704 \mathrm{E}-04$ \\
\hline 5 & $3.5192 \mathrm{E}-03$ & $1.3585 \mathrm{E}-02$ & $1.9022 \mathrm{E}-03$ & $3.6915 \mathrm{E}-03$ & $1.9139 \mathrm{E}-02$ & $1.9044 \mathrm{E}-03$ \\
\hline
\end{tabular}
numerical results with the analytical solutions and solutions in [10]. This method includes Thin Plate Splines (TPS) RBF collocation method [10]. We denote our scheme by IMQQI. The $\mathrm{L}_{2}, \mathrm{~L}_{\infty}$ and RMS error norms which are defined by

$$
\begin{gathered}
\mathrm{L}_{2}=\left\|\tilde{u}^{n}-u^{n}\right\|_{2}=\sqrt{h \sum_{j=0}^{N}\left(\check{u}^{n}\left(x_{j}\right)-u^{n}\left(x_{j}\right)\right)^{2}}, \\
\mathrm{~L}_{\infty}=\left\|\check{u}^{n}-u^{n}\right\|_{\infty}=\max _{0 \leqslant j \leqslant N}\left|\check{u}^{n}\left(x_{j}\right)-u^{n}\left(x_{j}\right)\right|, \\
\operatorname{RMS}=\sqrt{\left(\sum_{j=0}^{N}\left(\check{u}^{n}\left(x_{j}\right)-u^{n}\left(x_{j}\right)\right)^{2}\right) /(N+1)},
\end{gathered}
$$

Table 1. The comparison of the $\mathrm{L}_{\infty}, \mathrm{L}_{2}$ and RMS errors of our method with the results of [10] at different times of experiment 1 .

are used to measure the accuracy of our scheme where $\check{u}$ is the approximation solution. In all experiments, the shape parameter $s$ is considered twice of the shape parameter $c$. 
Table 2. The comparison of the $\mathrm{L}_{\infty}, \mathrm{L}_{2}$ and RMS errors of our method with the results of [10] at different times of experiment 2.

\begin{tabular}{|c|c|c|c|c|c|c|}
\hline \multirow[t]{2}{*}{$\mathrm{t}$} & \multicolumn{3}{|c|}{ IMQQI; $N=10$} & \multicolumn{3}{|c|}{ TPSM $; N=100$} \\
\hline & $\mathrm{L}_{\infty}$ & $\mathrm{L}_{2}$ & RMS & $\mathrm{L}_{\infty}$ & $\mathrm{L}_{2}$ & RMS \\
\hline 1 & $1.2590 \mathrm{E}-05$ & $2.0694 \mathrm{E}-05$ & $6.2397 \mathrm{E}-06$ & $1.2540 \mathrm{E}-05$ & $6.5422 \mathrm{E}-05$ & $6.5097 \mathrm{E}-06$ \\
\hline 3 & $1.5428 \mathrm{E}-05$ & $3.7065 \mathrm{E}-05$ & $1.1175 \mathrm{E}-06$ & $1.5554 \mathrm{E}-05$ & $1.1717 \mathrm{E}-04$ & $1.1659 \mathrm{E}-05$ \\
\hline 5 & $3.3625 \mathrm{E}-05$ & $6.9684 \mathrm{E}-05$ & $2.1010 \mathrm{E}-05$ & 3.3792E-05 & $2.2011 \mathrm{E}-04$ & 2.1902E-05 \\
\hline 7 & $3.7412 \mathrm{E}-05$ & 8.1943E-05 & $2.4706 \mathrm{E}-05$ & $3.7753 \mathrm{E}-05$ & 2.5892E-04 & 2.5763E-05 \\
\hline
\end{tabular}

The computations associated with our experiments are performed in Maple 18 on a PC with a CPU of 2.4 GHZ.

Experiment 1. Consider the Klein-Gordon equation (1) with $\alpha=-1, \beta=0, \gamma=1, k=2$ and $f(x, t)=6 x t\left(x^{2}-t^{2}\right)+$ $x^{6} t^{6}$ in interval $0 \leqslant x \leqslant 1$. The initial conditions are given by

$$
\begin{aligned}
& u(x, 0)=0, \quad 0 \leqslant x \leqslant 1, \\
& u_{t}(x, 0)=0, \quad 0 \leqslant x \leqslant 1 .
\end{aligned}
$$

The exact solution is given in [40] as

$$
u(x, t)=x^{3} t^{3} .
$$

The boundary function $g(x, t)$ can be extracted from the exact solution. The $\mathrm{L}_{2}, \mathrm{~L}_{\infty}$ and RMS errors in the solutions with $N=20, \Delta t=0.0001$ and $c=4.075 \times 10^{-2}$ that calculated in 50 points are listed in Table 1. Our numerical results are compared with the results in [10]. Moreover, graphs of exact and numerical solutions and error of method for different times are drawn in Fig. 1. It is observed from the Table 1 that the proposed method requires less nodes to attain the accuracy of the TPS method [10] and has better accuracy than TPS method [10].

Experiment 2. In this experiment, the Klein-Gordon equation (1) is considered with $\alpha=-1, \beta=0, \gamma=1, k=2$ and $f(x, t)=-x \cos (t)+x^{2} \cos (t)$ in interval $-1 \leqslant x \leqslant 1$. The initial conditions are given by

$$
\begin{aligned}
& u(x, 0)=x, \quad-1 \leqslant x \leqslant 1, \\
& u_{t}(x, 0)=0, \quad-1 \leqslant x \leqslant 1 .
\end{aligned}
$$

The exact solution is given in [40] as

$$
u(x, t)=x \cos (t) .
$$

\begin{tabular}{|c|c|c|c|c|c|c|}
\hline \multirow[t]{2}{*}{$\mathrm{t}$} & \multicolumn{3}{|c|}{ IMQQI; $N=50$} & \multicolumn{3}{|c|}{ TPSM $; N=200$} \\
\hline & $\mathrm{L}_{\infty}$ & $\mathrm{L}_{2}$ & RMS & $\mathrm{L}_{\infty}$ & $\mathrm{L}_{2}$ & RMS \\
\hline 1 & $4.2672 \mathrm{E}-05$ & $2.8456 \mathrm{E}-04$ & $2.0242 \mathrm{E}-05$ & $5.0705 \mathrm{E}-05$ & $2.9474 \mathrm{E}-04$ & $2.0789 \mathrm{E}-05$ \\
\hline 2 & 4.9397E-04 & $2.6266 \mathrm{E}-03$ & $1.8577 \mathrm{E}-04$ & $5.0260 \mathrm{E}-04$ & $2.7082 \mathrm{E}-03$ & $1.9102 \mathrm{E}-04$ \\
\hline 3 & $2.0380 \mathrm{E}-03$ & $9.5859 \mathrm{E}-03$ & $6.7016 \mathrm{E}-04$ & $2.0612 \mathrm{E}-03$ & $9.7246 \mathrm{E}-03$ & $6.8592 \mathrm{E}-04$ \\
\hline 4 & $6.4162 \mathrm{E}-03$ & $2.3457 \mathrm{E}-02$ & $1.8844 \mathrm{E}-03$ & $6.5720 \mathrm{E}-03$ & $2.7881 \mathrm{E}-02$ & $1.9666 \mathrm{E}-03$ \\
\hline 5 & $1.7941 \mathrm{E}-02$ & $7.4214 \mathrm{E}-02$ & $5.2391 \mathrm{E}-03$ & $1.9067 \mathrm{E}-02$ & 7.7337E-02 & $5.4549 \mathrm{E}-03$ \\
\hline
\end{tabular}
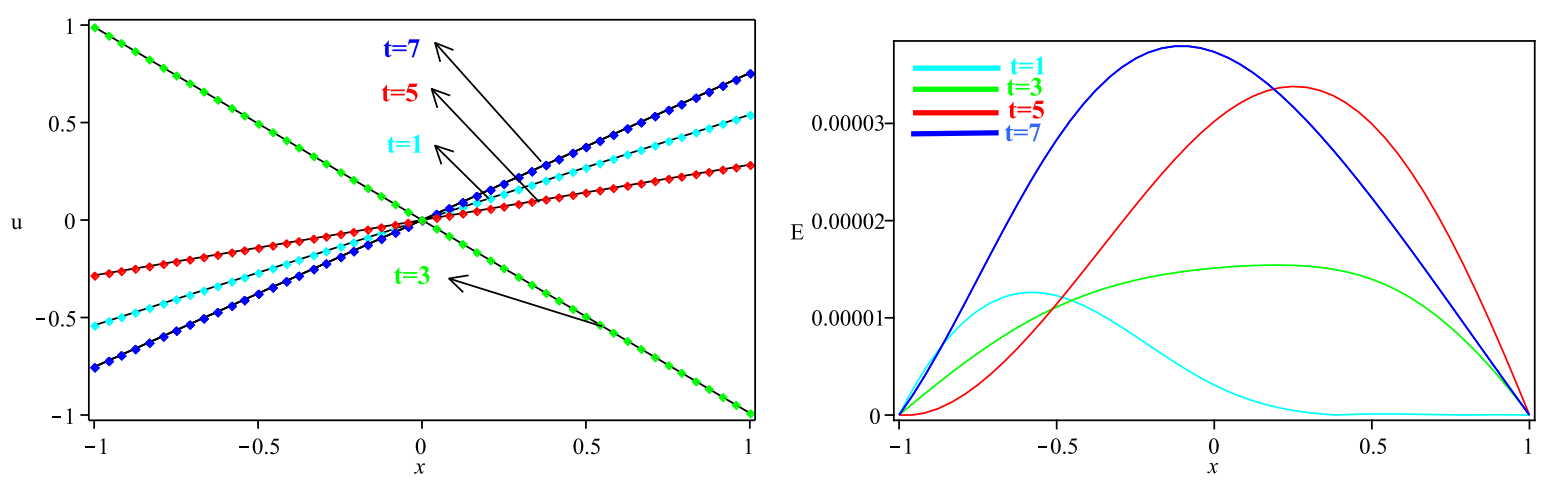

Figure 2. Exact and numerical solutions (left) and error of method (right) at different times with $\Delta t=0.0001$ and $N=10$ of experiment 2 .

Table 3. The comparison of the $\mathrm{L}_{\infty}, \mathrm{L}_{2}$ and RMS errors of our method with the results of [10] at different times of experiment 3 .

The boundary function $g(x, t)$ can be extracted from the exact solution. The $\mathrm{L}_{2}, \mathrm{~L}_{\infty}$ and RMS errors in the solutions with $N=10, \Delta t=0.0001, c=1.63 \times 10^{-2}$ that calculated in 100 points are listed in Table 2 and compared with the results 

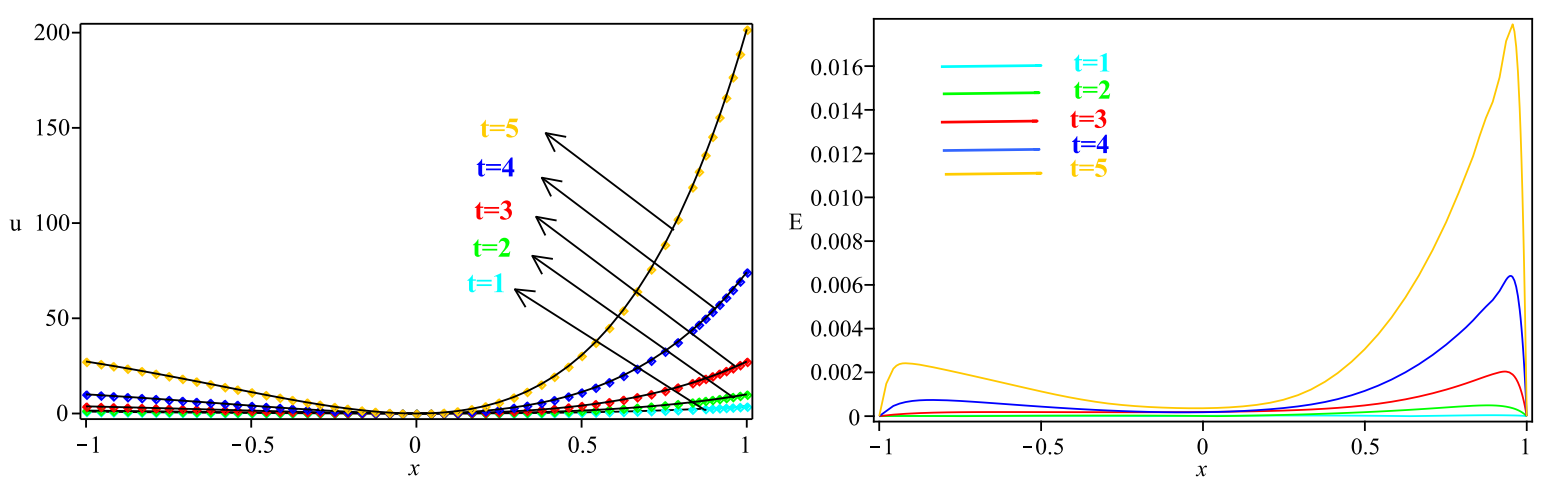

Figure 3. Exact and numerical solutions (left) and error of method (right) at different times with $\Delta t=0.001$ and $N=50$ of experiment 2 .
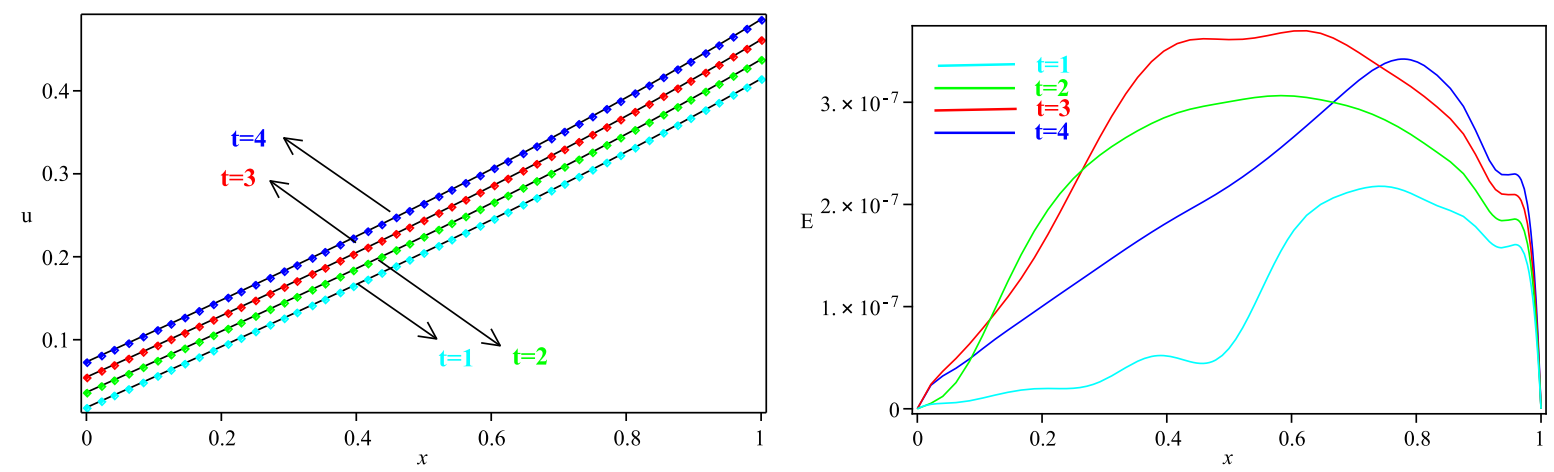

Figure 4. Exact and numerical solutions (left) and error of method (right) at different times with $\Delta t=0.001, N=20$ and $C=0.05$ of experiment 4 .

in [10]. The graphs of exact and numerical solutions and error of method for different times are given in Fig. 2 . Table 2 indicates that the proposed method requires less nodes to attain the accuracy of the TPS method [10]. Also, it show that this scheme performs better than TPS method.

Experiment 3. Consider the nonlinear Klein-Gordon equation (1) with $\alpha=-1, \beta=1, \gamma=1, k=3$ and $f(x, t)=$ $\left(x^{2}-2\right) \cosh (x+t)-4 x \sinh (x+t)+x^{6}(\cosh (x+t))^{3}$ in interval $-1 \leqslant x \leqslant 1$. The initial conditions are given by

$$
\begin{aligned}
& u(x, 0)=x^{2} \cosh (x), \quad-1 \leqslant x \leqslant 1, \\
& u_{t}(x, 0)=x^{2} \sinh (x), \quad-1 \leqslant x \leqslant 1 .
\end{aligned}
$$

The exact solution is given as

$$
u(x, t)=x^{2} \cosh (x+t)
$$

The boundary function $g(x, t)$ can be extracted from the exact solution. The $\mathrm{L}_{2}, \mathrm{~L}_{\infty}$ and RMS error norms in the solutions with $N=50, \Delta t=0.0001$ and $c=8.15 \times 10^{-2}$ that calculated in 200 points are shown in Table 3 . We compare our results with the results in [10]. The graphs of exact and numerical solutions and error of method for different times are presented in Fig. 3. Table 3 shows that the presented method requires less nodes to attain the accuracy of the TPS method [10].

Experiment 4. In this experiment, the Klein-Gordon equation (1) is considered with $\alpha=-2.5, \beta=1, \gamma=1.5, k=3$ and $f(x, t)=0$ in interval $0 \leqslant x \leqslant 1$. The initial conditions are given by

$$
\begin{aligned}
& u(x, 0)=B \tan (K x), \quad 0 \leqslant x \leqslant 1, \\
& u_{t}(x, 0)=B C K \sec ^{2}(K x), \quad 0 \leqslant x \leqslant 1 .
\end{aligned}
$$


Table 4. The comparison of the $\mathrm{L}_{\infty}, \mathrm{L}_{2}$ and RMS errors of our method with the results of [10] with $C=0.05$ at different times of experiment 4 .

\begin{tabular}{|c|c|c|c|c|c|c|}
\hline \multirow[t]{2}{*}{$\mathrm{t}$} & \multicolumn{3}{|c|}{ IMQQI; $\quad \mathrm{N}=20$} & \multicolumn{3}{|c|}{ TPSM; $\quad \mathrm{N}=100$} \\
\hline & $\mathrm{L}_{\infty}$ & $\mathrm{L}_{2}$ & RMS & $\mathrm{L}_{\infty}$ & $\mathrm{L}_{2}$ & RMS \\
\hline 1 & $2.1781 \mathrm{E}-07$ & $1.2850 \mathrm{E}-06$ & $1.2787 \mathrm{E}-07$ & $3.6497 \mathrm{E}-07$ & $1.7861 \mathrm{E}-06$ & 1.7772E-07 \\
\hline 2 & $3.0648 \mathrm{E}-07$ & $1.4099 \mathrm{E}-06$ & $1.3980 \mathrm{E}-07$ & $3.8952 \mathrm{E}-07$ & $1.5383 \mathrm{E}-06$ & $1.5306 \mathrm{E}-07$ \\
\hline 3 & $3.7008 \mathrm{E}-07$ & 1.3144E-06 & $1.7004 \mathrm{E}-07$ & 4.2123E-07 & $1.7275 \mathrm{E}-06$ & 1.7190E-07 \\
\hline 4 & $3.4230 \mathrm{E}-07$ & $2.0012 \mathrm{E}-06$ & $1.2002 \mathrm{E}-07$ & $4.5928 \mathrm{E}-07$ & $2.0097 \mathrm{E}-06$ & $1.9997 \mathrm{E}-07$ \\
\hline
\end{tabular}
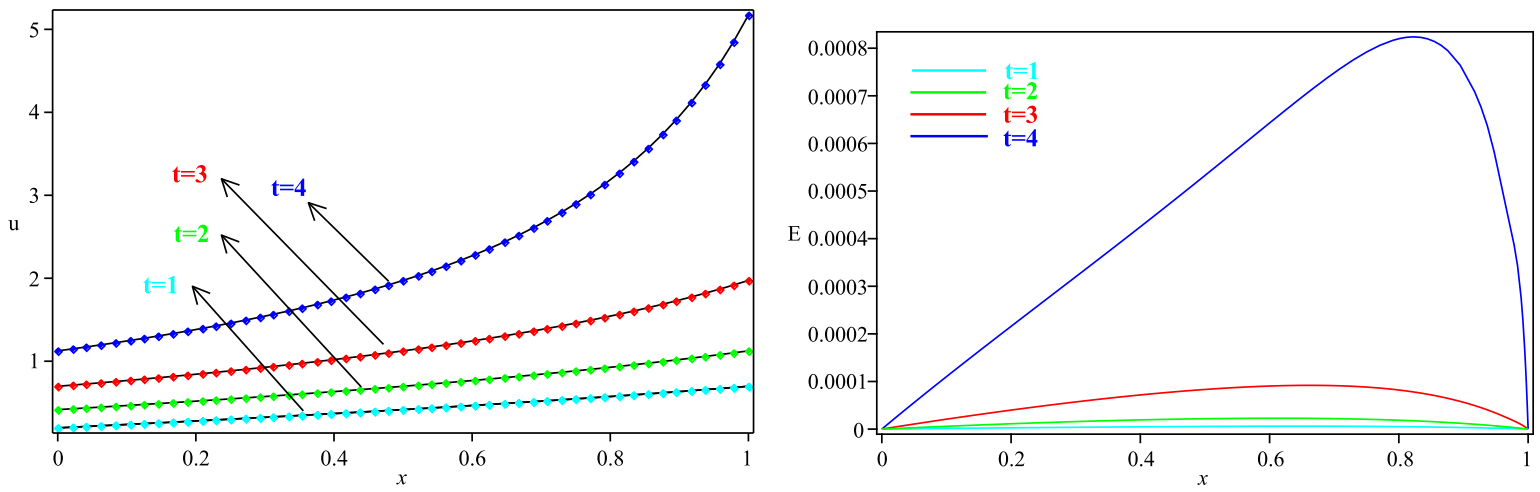

Figure 5. Exact and numerical solutions (left) and error of method (right) at different times with $\Delta t=0.001, N=50$ and $C=0.5$ of experiment 4 .

Table 5. The comparison of the $\mathrm{L}_{\infty}, \mathrm{L}_{2}$ and RMS errors of our method with the results of [10] with $C=0.5$ at different times of experiment 4 .

\begin{tabular}{|c|c|c|c|c|c|c|}
\hline \multirow[t]{2}{*}{$\mathrm{t}$} & \multicolumn{3}{|c|}{ IMQQI; $N=50$} & \multicolumn{3}{|c|}{ TPSM; $N=100$} \\
\hline & $\mathrm{L}_{\infty}$ & $\mathrm{L}_{2}$ & RMS & $\mathrm{L}_{\infty}$ & $\mathrm{L}_{2}$ & RMS \\
\hline 1 & $5.2134 \mathrm{E}-06$ & $4.0608 \mathrm{E}-05$ & $4.0392 \mathrm{E}-06$ & $5.9964 \mathrm{E}-06$ & $4.0761 \mathrm{E}-05$ & $4.0559 \mathrm{E}-06$ \\
\hline 2 & $2.1805 \mathrm{E}-05$ & $1.5588 \mathrm{E}-04$ & $1.5505 \mathrm{E}-05$ & 2.1973E-05 & $1.5769 \mathrm{E}-04$ & $1.5691 \mathrm{E}-05$ \\
\hline 3 & $9.0113 \mathrm{E}-05$ & $6.3231 \mathrm{E}-04$ & $6.5903 \mathrm{E}-05$ & $9.0893 \mathrm{E}-05$ & $6.4792 \mathrm{E}-04$ & $6.4470 \mathrm{E}-05$ \\
\hline 4 & $8.2372 \mathrm{E}-04$ & $5.3386 \mathrm{E}-03$ & $5.3219 \mathrm{E}-04$ & $8.2945 \mathrm{E}-04$ & $5.3572 \mathrm{E}-03$ & $5.3306 \mathrm{E}-04$ \\
\hline
\end{tabular}

The exact solution is given in [15] as

$$
u(x, t)=B \tan (K(x+C t))
$$

where $B=\sqrt{\frac{\alpha}{\beta}}$ and $K=\sqrt{\frac{\alpha}{-2 \mu+C^{2}}}$. The boundary function $g(x, t)$ can be extracted from the exact solution. The $\mathrm{L}_{2}, \mathrm{~L}_{\infty}$ and RMS errors in the solutions with $N=20, N=50, \Delta t=0.001$ for $C=0.05$ and $C=0.5$ are tabulated in Tables 4 and 5 , respectively. The shape parameter $c$ is chosen as $4.075 \times 10^{-2}$ and $1.63 \times 10^{-2}$ for $N=20$ and $N=50$, respectively. The graphs of exact and numerical solutions and error of method for different times are drawn in Figs. 4 and 5.

Tables 4 and 5 show that the proposed method requires less nodes to attain the accuracy of the TPS method [10].

\section{Conclusion}

In this paper, a mixed method of high accuracy multiquadric quasi-interpolation scheme and integrated radial basis function networks scheme is used to obtain approximate solutions of the nonlinear Klein-Gordon equation. Also, the order of nonlinearity of the problem is quadratic and cubic. In discretization of the temporal derivative, we employed the $\theta$-weighted scheme. The numerical results which is given in the previous section demonstrate the good accuracy of the presented method. Tables and Figures of the solved experiments indicate that this scheme requires less nodes to attain accuracy of TPS method [10].

\section{REFERENCES}

[1] A. Aminataei, M. Sharan, Using multiquadric method in the numerical solution of ordinary differential equations with a singularity point and partial differential equations in one and two dimenions, Euro. J. Scien. Res., 10(2) (2005) 19-45.

[2] A. Aminataei, M.M. Mazaraei, Numerical solution of Poisson's equation using radial basis function networks on the polar coordinate, Comput. Math. Appl., 56(11) (2008) 2887-2895.

[3] A. Aminataei, M. Sarboland, Numerical solution of two-dimensional shrodinger equation using multivariate quasi-interpolation scheme, The 44th Annual Iranian Mathemathics Conference, Ferdowsi Univ. Of Mashhad, pp. 732-735, 27-30 Aug. 2013.

[4] A. Aminataei, Z. Soori, A fourth-order compact finite difference-spectral and spectral method for the solution of sine-Gordon equation, Pensee J., 75 (12) (2013) 33-56. 
[5] R.K. Beatson, M.J.D. Powell, Univariate multiquadric approximation: quasi-interpolation to scattered data, Constr. Approx., 8 (3) (1992) 275-288.

[6] R.H. Chen, Z.M. Wu, Solving hyperbolic conservation laws using multiquadric quasi-interpolation, Numer. Methods Partial Differential Equations, 22 (4) (2006) 776-796.

[7] R.H. Chen, Z.M. Wu, Solving partial differential equation by using multiquadric quasi-interpolation, Appl. Math. Comput., 186 (2) (2007) 1502-1510.

[8] M. Dehghan, A. Shokri, A numerical method for KdV equation using collation and radial basis functions, Nonlinear Dyn., 50 (2007) 111-120.

[9] M. Dehghan, A. Shokri, A numerical method for solution of the two-dimensional sine-Gordon equation using the radial basis functions, Mathematics and Computers Simulation, 79 (2008) 700-715.

[10] M. Dehghan, A. Shokri, Numerical solution of the nonlinear Klein-Gordon equation using radial basis functions, J. Comput. Appl. Math., 230 (2009) 400-410.

[11] S.M. El-Sayed, The decomposition method for studying the Klein-Gordon equation, Chaos Solitons Fractals, 18 (2003) $1025-1030$.

[12] Y.C. Hon, X.Z. Mao, An efficient numerical scheme for Burgers' equation, Appl. Math. Comput., 95 (1998) 37-50.

[13] Z.W. Jiang, R.H. Wang, C.G. Zhu, M. Xu, High accuracy multiquadric quasi-interpolation, Appl. Math. Modelling, 35 (2011) $2185-$ 2195.

[14] Z.W. Jiang, R.H. Wang, Numerical solution of one-dimensional sine-Gordon equation using high accuracy multiquadric quasiinterpolation, Appl. Math. comput., 218 (2012) 7711-7716.

[15] D. Kaya, S.M. El-Sayed, A numerical solution of the Klein-Gordon equation and convergence of the decomposition method, Appl. Math. Comput., 156 (2004) 341-353.

[16] M. Lakestani, M. Dehghan, Collocation and finite difference-collocation methods for the solution of nonlinear Klein-Gordon equation, Comput. Phys. Commun. 181 (8) (2010) 1392-1401.

[17] N. Mai-Duy, T. Tran-Cong, Numerical solution of differential equations using multiquadric radial basis function networks, Neural Netw., 14 (2001) 185-199.

[18] M.M. Mazaraei, A. Aminataei, Numerical solution of elliptic partial differential equations using direct and indirect radial basis function networks, Euo-Asian J. Appl. Sci., 2(2) (2005) 24-32.

[19] M.M. Mazaraei, A. Aminataei, Numerical solution of elliptic partial differential equations using radial basis function networks and comparison between RBFN and Adomain method, Far East J. Appl. Math., 32(1) (2008) 113-126.

[20] M.M. Mazaraei, A. Aminataei, Numerical solution of Poisson's equation using a combination of logarithmic and multiquadric radial basis function networks, J. of Appl. Maths., 2012, Article ID 286391, 13 pages, 2012. doi:10.1155/2012/286391.

[21] J.K. Perring, T.H. Skyrme, A model unified field equation, Nucl. Phys. 31 (1962) 550-555.

[22] M. Sarboland, A. Aminataei, Solving partial differential equations by meshless Petrov-Galerkin method combined with radial basis functions and Lagrange polynomials, J. Math. Sci. Adv. Appl., 1(2) (2008) 291-298.

[23] M. Sarboland, A. Aminataei, Meshless Petrov-Galerkin method by using radial basis functions in the numerical solution of partial differential equations, ICASTOR J. of Math. Scis., 3(1) (2009) 33-39.

[24] M. Sarboland, A. Aminataei, Multiquadric quasi-interpolation scheme for solving the two-dimensional Burgers' equation, The 43rd Annual Irannian Maths. Conf., Univ. of Tabriz, pp. 781-784, 27-30 Aug. 2012.

[25] M. Sarboland, A. Aminataei, Taylors collocation method for the numerical solution of the two-dimensional Burgers' equations using a combination of logarithmic and multiquadric radial basis function networks, The 5th maths. Conf. of Payame Noor Univ., 24-25 Oct. 2012-Iran-Shiraz, 442-445.

[26] M. Sarboland, A. Aminataei, Taylor's meshless Petrov-Galerkin method for the numerical solution of Burgers' equation by radial basis functions, ISRN Appl. Maths., 2012, Article ID 254086, 15 pages, doi:10.5402/2012/254086.

[27] M. Sarboland, A. Aminataei, On the numerical solution of one-dimensional nonlinear nonhomogeneous Burgers' equation, J. Appl. Math., 2014, 15 pages, doi:10.1155/2014/598432.

[28] M. Sarboland, A. Aminataei, Numerical solution of the two-dimensional sine-Gordon equation using multivariate multiquadric quasiinterpolation scheme, Istanbul, Turkey, ICMS, 9-11, July 2014, p. 223.

[29] M. Sarboland, A. Aminataei, Solving nonlinear Klien-Gordon equation with high accuracy multiquadric quasi-interpolation scheme, J. Appl. Func. Anal., 9(1-2) (2014) 132-143.

[30] M. Sarboland, A. Aminataei, The dual reciprocity boundary element method for two-dimensional Burgers' equations with inverse multiquadric approximation scheme, J. Conc. Appli. Maths., 12(1-2) (2014) 102-115.

[31] M. Sarboland, A. Aminataei, Improvment of the multiquadric quasi-interpolation $\mathcal{L}_{\mathcal{W}_{2}}$, J. Math. Compu. Sci., 11 (2014) 13-21. 
[32] M. Sarboland, A. Aminataei, An efficient numerical scheme for coupled nonlinear Burgers' equations, Appl. Math. Inf. Sci., 9(1) (2015) 245-255.

[33] M. Sarboland, A. Aminataei, On the numerical solution of nonlinear Korteweg-de Vries equation, Sys. Sci. \& Cont. Eng., 3 (2015) 69-80, http://dx.doi.org/10.1080/21642583.2014.986340.

[34] Sirendaoreji, Auxiliary equation method and new solutions of Klein-Gordon equations, Chaos Solitons Fractals, 31(4) (2007) 943950.

[35] Sirendaoreji, A new auxiliary equation and exact travelling wave solutions of nonlinear equations, Phys. Lett. A, 356 (2) (2006) 124-130.

[36] Z. Soori, A. Aminataei, A well-posedness of the sine-Gordon equation using homotopy perturbation method, The 43rd Annual Iranian Mathemathics Conference, Univ. of Tabriz, pp. 743-769, 27-30 Aug. 2012.

[37] Z. Soori, A. Aminataei, The spectral method for solving sine-Gordon equation using a new orthogonal polynomial, ISRN Applied Mathematics, 2012, Article ID 462731, 12 pages, doi: 10.5402/2012/462731.

[38] R. Temam, Infinite-Dimensional Dynamical Systems in Mechanics and Physics, Applied Mathematical Sciences, vol. 68, SpringerVerlag, 1988.

[39] S.K. Vanani, A. Aminataei, On the numerical solution of delay differential equation using multiquadric approximation scheme, Int. J. Func. Diff. Eqn., 17(3-4) (2010) 391-399.

[40] A.M. Wazwaz, The modified decomposition method for analytic treatment of differential equations, Appl. Math. Comput., 173 (2006) $165-176$.

[41] A.M. Wazwaz, The tanh and the sine-cosine methods for compact and non-compact solutions of the nonlinear Klein-Gordon equation, Appl. Math.Comput., 167 (2005) 1179-1195.

[42] A.M. Wazwaz, New travelling wave solutions to the Boussinesq and the Klein-Gordon equations, Communications in Nonlinear Science and Numerical Simulation, 13 (2008) 889-901.

[43] Z.M. Wu, R. Schaback, Shape preserving properties and convergence of univariate multiquadric quasi- interpolation, Acta. Math. Appl. Sinica (English Ser.), 10 (4) (1994) 441-446.

[44] Z.M. Wu, Dynamically knot and shape parameter setting for simulating shock wave by using multiquadric quasi-interpolation, Engineering Analysis with Boundary Elements, 29 (2005) 354-358.

[45] M.L. Xiao, R.H. Wang, C.H. Zhu, Applying multiquadric quasi-interpolation to solve KdV equation, Mathematical Research Exposition, 31 (2011) 191-201. 